\title{
Context matching and judgments of recency
}

\author{
DOUGLAS L. HINTZMAN \\ University of Oregon, Eugene, Oregon
}

\begin{abstract}
An experiment was done to test a context-matching explanation of memory for recency under steady-state conditions. Subjects went through a list of 550 names, in which individual names were repeated at lags of 5-30 other items. The names were shown in two different styles or contexts. An old versus new recognition decision was made on each name, and each old response was followed by a numerical judgment of recency (JOR). When first- and second-presentation contexts were the same, recognition hit rates were higher, and mean JORs were shorter (more recent), than when the two contexts were different. The JOR result is as predicted by the context-matching hypothesis.
\end{abstract}

When asked at what time in the past a remembered event occurred, people usually try to infer the answer by relating the event to other facts and, ultimately, to dates or temporal landmarks (Friedman, 1993). Underlying such strategies, however, there seems to be a raw, nonanalytic sense of a memory's age. Although poorly suited for fine-grained temporal discrimination, this recency sense is highly accurate for the discrimination of different orders of magnitude. For example, a normal person would never think that a remembered event of a minute ago may have happened yesterday or that an event experienced yesterday may have occurred a year in the past. ${ }^{1}$

Theorists have proposed several kinds of mechanisms to explain the intuitive ability to judge a memory's age. The pioneering scientist Robert Hooke proposed that the soul interacted with the brain at a single anatomical location, where it continually formed new memories and added them to a chain. Each new memory displaced the entire chain outward, in a coil that filled a larger and larger brain volume with the passage of time. Because newer memories were closer to the location of the soul's interaction than older memories were, the soul could use the radial distance to a reactivated memory to determine its age (Hooke, 1705/1969). Although linear rather than coiled, Murdock's (1974) conveyor-belt model may be seen as a variation on Hooke's idea. The Gestalt psychologists, too, hypothesized that memory was inherently temporally ordered (Koffka, 1935; Köhler, 1929), without specifically considering how a memory's age or recency might be retrieved.

Closer to the mainstream is the hypothesis that recency judgments are based on strength (Hartley, 1834; Hinrichs, 1970). ${ }^{2}$ A problem with this idea is that strength is usually assumed to be strongly influenced by repetition, and judgments of recency (JORs) typically are affected very

Correspondence concerning this article should be addressed to D. L. Hintzman, Department of Psychology, University of Oregon, Eugene, OR 97403 (e-mail: hintzman@oregon.uoregon.edu). little by repetition. The most consistent effects of repetition seem to occur when presentations are massed rather than spaced (Flexser \& Bower, 1974; Peterson, 1967; Peterson, Johnson, \& Coatney, 1969)—a situation that yields the poorest long-term learning. A solution to the recencyversus-repetition dilemma is to propose that recency judgments are based on strength in short-term memory (Konorski, 1961) and measures such as frequency judgments are based on strength in long-term memory (Hintzman, 1969). But the ability to judge recencies extends too far into the past to be spanned by the short-term store, as it is traditionally conceived in cognitive psychology. Wickelgren (1974) proposed that memory for recency was based on the fragility of the retrieved memory - that is, the degree to which the memory had not yet been consolidated. This process could have the temporal dynamics necessary to explain a general sense of recency. Like radial distance in Hooke's (1705/1969) theory, consolidation could continue growing long after initial encoding of the event.

In discussing their pioneering experiments on judgments of relative recency, Yntema and Trask (1963) attributed the ability to mnemonic time tags. This term suggests a dedicated representation of time but is otherwise ambiguous. A time tag could be analogous to a reading on a stop watch, which is reset to zero each time a particular stimulus occurs. Neurons in the temporal lobe of the monkey that might serve this function have been reported by Xiang and Brown (1998). Alternatively, a time tag could be analogous to a calendar-and-clock reading. A model by Brown, Preece, and Hulme (2000) postulates a bank of hierarchically ordered neural oscillators with a wide range of periodicities. The states of the oscillators are a salient part of an event's context and are incorporated into the memory trace. To explain recency judgments, Brown et al. propose that oscillator states retrieved from memory are compared with the oscillator states at the time that retrieval takes place.

But context could mediate JORs without incorporating a time tag per se. At the end of their Experiment 3, Hintzman, Block, and Summers (1973) surprised their subjects 
with a test of the ability to assign words to a previous list (List 1,2, or 3) and to a within-list position (near the beginning vs. near the end). The judgments showed that list and within-list position were remembered with a certain degree of independence - that is, the subjects tended to remember the within-list position correctly even when they got the list membership wrong. Crucially, in one condition the judgment test was given immediately after List 3, and in another it was delayed by about $15 \mathrm{~min}$. Accuracy was essentially unaffected by the increased retention interval, except for the ability to assign words to the end of List 3. Hintzman et al. suggested a context-matching mechanism to explain this result. If contextual elements tend to drift over time (e.g., Bower, 1972; Estes, 1955), the degree to which the retrieved context matches the current context could mediate a judgment of the test item's recency. Such information would especially benefit judgments for items from the end of List 3 on an immediate test. Context matching could provide what Friedman (1993) called distance information, because the degree of match declines as the event drifts into the past.

Further support for the context-matching hypothesis comes from an experiment that eliminated useful temporal landmarks by having subjects make JORs in a long, runningrecognition list (Hintzman, 2001, Experiment 2). Subjects saw a 500-word list, within which items were repeated after lags of 10-80 other words. When a word occurred, the subjects first judged whether it was old or new. If they judged the word to be old, they also (1) rated whether they remembered details of the word's original occurrence or the word just seemed familiar, and (2) gave a numerical JOR. The JORs subjects gave to words that they said they remembered were quantitatively quite different from those they gave to words that they rated as familiar. At every lag, the subjects judged that remembered items had occurred more recently than familiar items. This difference was much greater at short lags than at long lags. Nevertheless, JORs to familiar items were more accurate than chance. Moreover, the two JOR functions overlapped. That is, JORs for familiar items at lag 10 were shorter than they were for remembered items at lag 80 .

To relate these findings to the context-matching hypothesis, we can assume that retrieved contextual information underlies both remember and familiarity ratings but that remember ratings identify memories that are rich in contextual information and familiarity ratings identify memories that are relatively poor in contextual information. This is consistent with signal detection models of remember versus know ratings (Donaldson, 1996; Hirshman \& Henzler, 1998; Hirshman \& Master, 1997; Inoue \& Bellezza, 1998), assuming that the decision axis reflects the amount of list-relevant context that is retrieved. Now, if apparent recency increases with the number of retrieved contextual elements that match the current context, sensitivity to recency will be greater for remembered items than for familiar items. Nevertheless, remembered items will be judged to be more recent than familiar items even at the longest lags, because remembered items have more retrieved context that can be matched against the current context (Hintzman, 2001).

The foregoing arguments are ad hoc, and a direct test of the context-matching hypothesis is needed. The main goal of the present study was to find an aspect of context that can be explicitly manipulated independently of time, that subjects have to process, and that is incidental to the JOR task. The prediction is that JORs will be shorter when study and test contexts match than when the contexts are different. Because context manipulations can also influence whether a test item is recognized as old, JORs were made contingent on a positive recognition response. An additional goal was to eliminate the use of temporal landmarks, such as the beginning of a list. The task was a combination of running recognition (Shepard $\&$ Teghtsoonian, 1961) and JOR. To establish steady-state conditions lacking in temporal landmarks, the repetition lags ranged from 5 to 30 items in a 550-item list (see Hinrichs \& Buschke, 1968; Hintzman, 2001; Yntema \& Trask, 1963). Stimulus materials were common first names, and the manipulated context was the name's physical appearance. (Examples of the two contexts that were used are shown in Figure 1.) When a name was repeated, the second-presentation context was different from the firstpresentation context on $50 \%$ of the trials.

\section{METHOD}

\section{Subjects}

A total of 38 University of Oregon students, all native speakers of English, participated for course credit. They were tested individually in sessions lasting less than $50 \mathrm{~min}$.

\section{Materials}

The stimuli were common female and male first names taken from a 1990 U.S. Census Web site. Initial lists of 500 male names and 500 female names were edited to remove close variants and names that appeared to be highly similar. This yielded a final list of 504 names, half male and half female. The names were displayed in two different contexts. In one, the name was shown in bold uppercase 24-point Helvetica font, flanked above and below by rows of plus signs. In the other, the name appeared in italic uppercase 48point Times font, with no flanking stimuli. Characters were black on a white background. Figure 1 shows examples.

\section{ELEANOR}

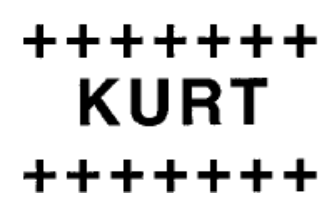

Figure 1. Examples of the experimental materials. 


\section{Procedure}

Each subject was seated in front of a Macintosh IIci computer controlling a full-page, gray-scale monitor. The subjects were told that they would see a long list of first names, in which many names would be repeated. Their first task was to decide whether each name was new or old in the list by responding on the $\mathrm{Z}$ or ?/ key of the keyboard, respectively. If they decided a name was old, they would also be asked to judge how far back in the list the name had occurred previously. They were told that no name would occur more than two times and that repetitions could occur after 5, 10, 15, 20, 25 , or 30 intervening names. The JORs, or lag judgments, were to be made using the 1-6 keys of the numerical keypad, which had been relabeled with the appropriate numbers.

The subjects were also shown examples of the two fonts or contexts that would be used to display the names. It was explained that the style in which a name appeared the second time could either match or mismatch the style it had appeared in the first time: "We want you to ignore the font style in making your judgments. That is, respond based on whether the name itself is old or new, regardless of its physical appearance." All the subjects conf irmed that they understood the instructions.

The list of names was 550 items long. ${ }^{3}$ The experimental program stepped through positions $P=1-550$, in sequence. It began by randomly selecting a name from the master list, choosing one of six repetition lags at random ( $\operatorname{lag}=5,10,15,20,25$, or 30), and assigning the name to both $P=1$ and $P=1+\operatorname{lag}$. For $P=2-550$, the program first checked to see whether an old name had already been assigned to $P$. If not, a new name was assigned to $P$, one of the six values of lag was chosen at random, and the second presentation of the name was assigned to $P+l a g$. If $P+l a g$ was already occupied by an old name, a new value of lag was randomly generated. If this second attempt failed to select an unoccupied value of $P+$ $l a g$, the name was not repeated in the list. One of the two contexts was selected at random for the first presentation, and the same was done independently for the second presentation. Data were analyzed only for test positions 32-550. This scheme resulted in a mean of 20.8 observations per condition (lag $\times$ same vs. different) per subject.

A 500-msec blank interval preceded the appearance of each test name. When the name appeared, the subject first indicated whether it was old or new. The response alternatives $(\mathrm{Z}=$ new and $? /=$ old $)$ were displayed on the screen in a single line about $1.5 \mathrm{~cm}$ beneath the stimulus itself. If the subject responded old, this instruction was immediately replaced by the two lines "How many items back?" and "5 1015202530 ." Each display remained on the screen until the subject responded. Illegal keypresses were followed by a warning and a repeated request for a response. The subjects were told to go through the list at their own pace. This typically took between 25 and $40 \mathrm{~min}$, roughly $3 \mathrm{sec}$ per item on average.

\section{RESULTS AND DISCUSSION}

One subject, who complained about the difficulty of the task, recorded by far the worst recognition and JOR performances. Another subject's cell phone rang during the experiment. Both subjects' data were dropped from further analysis, leaving an $N$ of 36.

Figure 2 shows the mean recognition hit proportions as a function of lag and of whether the first- and secondpresentation contexts were the same or different. Hit rates averaged .857 . For comparison, the mean false alarm rate was .055. The graph suggests a slight advantage of short over long lags and of same context over different context, both of which are consistent with previous recognition memory results. The hit rates were evaluated with orthogonal contrasts, using the $t$ test method outlined by Rosenthal and Rosnow (1985, pp. 56-62). Three contrasts were computed: one for linear trend on lag $[t(35)=$ $2.895, p<.01]$, one for same versus different context

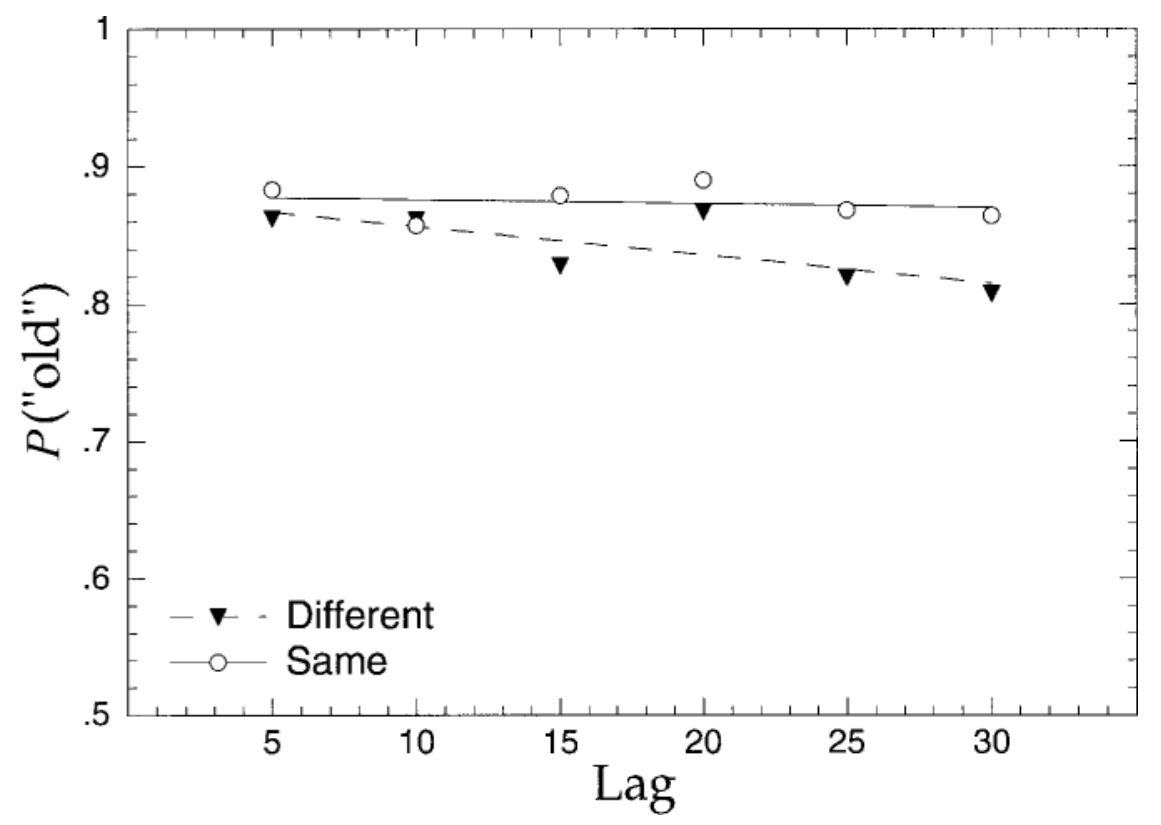

Figure 2. Probability of recognition [ $p$ (“old")] as a function of same versus different context and lag. 
$[t(35)=4.838, p<.001]$, and one for the lag $\times$ context interaction $[t(35)=2.110, p<.05]$. A ceiling effect may have contributed to the moderately significant interaction; however, it does suggest that context matching affected recognition more at the longer lags.

Figure 3 shows the mean JORs (or judged lag) for old items that were correctly identified as old. For comparison, with these values, the mean JOR following false alarms to new items was 21 . The means show a large effect of lag and a small but consistent effect of context. When the test context was different from the original context, names were judged to have occurred 0.61 items further back in the list (about $2 \mathrm{sec}$ earlier) than when the two contexts were the same. The JOR data were evaluated with the same three contrasts as before. The linear trend on lag was, of course, highly significant $[t(35)>$ $24]$. The effect of context was reliable $[t(35)=5.204$, $p<.001]$, but showed no tendency to interact with lag $[t(35)<1]$.

Given that context matching affects JORs, one might expect consistently greater accuracy in the same-context condition than in the different-context condition, but this was not the case. To see this, notice that the numbers on the abscissa and ordinate of Figure 3 are the same (lag and judged lag). Although same-context JORs tended to be more accurate than different-context JORs at lags 5 and 10, they were less accurate at lags 20 and above. It might be tempting to describe the effect of context on JORs as a shift in bias, but it is not a response bias, as this concept is usually understood. The effect depends on retrieval—specifi- cally, on the degree to which the cue and the memory trace match.

A possible explanation of the effect of context on mean JOR is that it is a side effect of the influence of context on recognition. The subjects were not allowed to withdraw a recognition decision after it had been made. Upon reflection, they may have sometimes decided that their old responses were wrong. The recognition rates in Figure 2 suggest that this might happen more often in the differentcontext condition than in the same-context condition, because recognition was generally less accurate when the contexts did not match. If subjects are forced to make a JOR to a name that they now believe to be new, they might be biased to respond with a long JOR.

This account can be evaluated by reference to Figure 4, which shows the distributions of JOR values collapsed over subjects. The upper left panel shows JORs following false alarms (old responses to new items), and these were indeed biased toward high values. If "recognition regret" were more common for different-context items than for same-context items and if it led to JORs similar to those for false alarms, differences between same- and different-context JORs should fall differentially at the highest JORs. The remaining panels of Figure 4 show that this was not the case. Differences were as pronounced at JOR $=5$ as they were at JOR $=25$ and 30 . Thus, context matching shifted the entire distributions of JORs.

The experiment shows that apparent recency is not determined solely by a property of the memory trace, as was

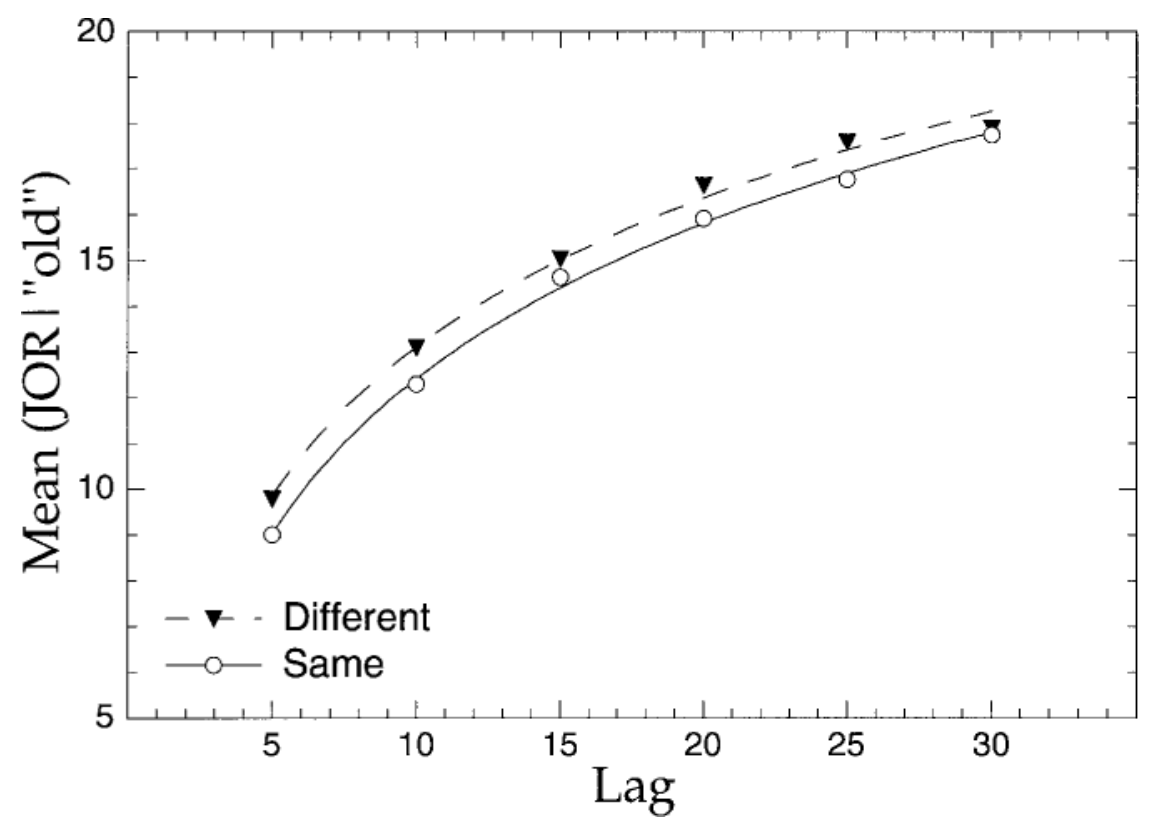

Figure 3. Mean judgment of recency (JOR) for correctly recognized old items, as a function of same versus different context and lag. Basing error on the subject $\times$ condition interaction, the $95 \%$ confidence intervals are mean \pm 0.51 . 

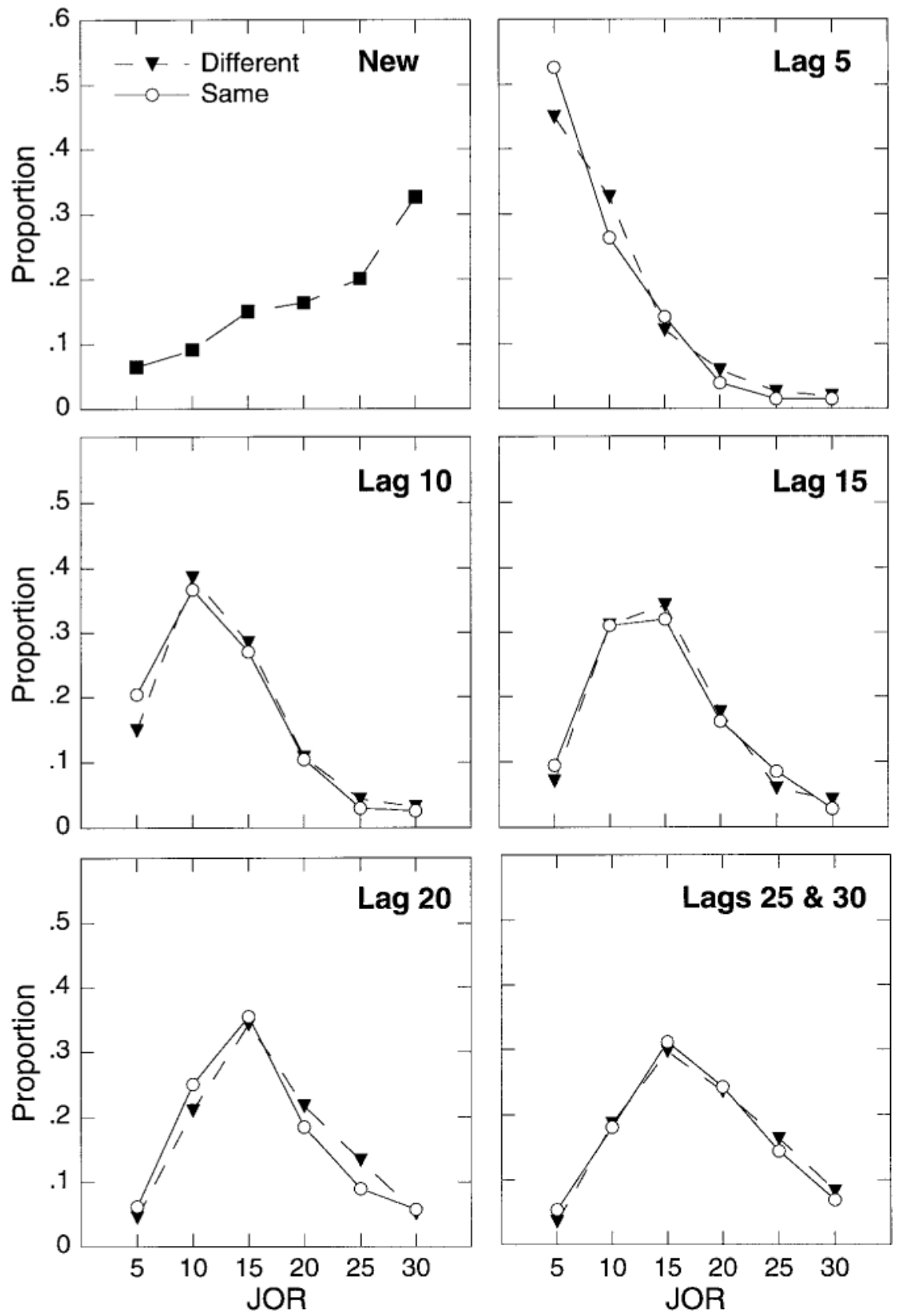

Figure 4. Judgment of recency (JOR) distributions for new items falsely recognized as old and for correctly recognized old items tested at lags 5-30.

predicted by several of the theories discussed earlier. Rather, it is influenced by the degree to which the original experience is reinstated by properties of the retrieval environment that are incidental to the subject's task. There seem to be two ways this could occur. One is that contextual features are retrieved from the memory trace and matched against those present in the retrieval environment, as was proposed by Hintzman et al. (1973). The other explanation is that the retrieval cue activates the trace in proportion to trace-cue similarity, which is de- termined partly by contextual features, and that trace activation, in turn, determines the JOR.

The second interpretation appears to be consistent with the effect of context matching on old-new recognition. However, recognition judgments and JORs cannot both be based on a single scalar quantity, such as trace activation. This point is made most simply by noting that context had a greater effect than did lag on recognition hit rate (Figure 2), whereas lag had a greater effect than did context on JOR (Figure 3). There is, thus, no monotonic func- 
tion by which one can predict JOR from recognition and vice versa (Bamber, 1979). If trace activation underlies both recognition and JOR, it must be weighted differently, or used in different ways, in the two judgment tasks.

A primary goal of this experiment was to obtain a large number of JORs from each subject under steadystate conditions that eliminate the use of temporal landmarks. The range of lags was consequently quite limited (5-30 items, or about 15-90 sec). Thus, although the data are consistent with the context-matching hypothesis, they raise the obvious question of whether the proposed mechanism would scale upward by orders of magnitude, from seconds through hours, days, months, and years. Conducting an adequately controlled study of the role of context in recency judgments over very long intervals would be a difficult challenge. One complicating factor is the need to control for failures to remember the target event. Another is the tendency of subjects to try to reason out an answer when a variety of associations can be recalled (Friedman, 1993).

Of course, intuitive judgments of the age of an event could be based on one mechanism at short intervals and a different mechanism or mechanisms at long intervals. This raises the question of whether context matching alone could underlie the sense of recency over time spans ranging from seconds to years, which in turn raises the question of how context is to be conceived. Bower (1972) described context as a kind of "internal monologue" in which the experimental subject describes "the free flow of the 'stream of consciousness." 'In addition to environmental and internal cues, it may include thoughts on the nature of the experimental task, the experimenter, the subject's performance, and the subject's purpose in volunteering for the experiment (p. 93).

James (1890) famously said of the stream of consciousness, "like Heraclitus ... we never descend twice into the same stream." He illustrated his point as follows:

Often we are ourselves struck at the strange differences in our successive views of the same thing. We wonder how we ever could have opined as we did last month about a certain matter. We have outgrown the possibility of that state of mind, we know not how. From one year to another we see things in new lights. What was unreal has grown real, and what was exciting is insipid. The friends we used to care the world for are shrunken into shadows; the women, once so divine, the stars, the woods, and the waters, how now so dull and common; the young girls that brought an aura of infinity, at present hardly distinguishable existences; the pictures so empty; and as for the books, what was there to find so mysteriously significant in Goethe, or in John Mill, so full of weight?... But what here strikes us so forcibly on the flagrant scale exists on every scale, down to the imperceptible transition from one hour's outlook to that of the next. Experience is remoulding us every moment, and our mental reaction on every given thing is really a resultant of our experience of the whole world up to that date. (James, 1890, pp. 233-234)

James's observations make it seem at least plausible that context matching could mediate apparent recency over a broad range of times.

\section{REFERENCES}

BAmber, D. (1979). State-trace analysis: A method of testing simple theories of causation. Journal of Mathematical Psychology, 19, 137-181.

BowER, G. H. (1972). Stimulus-sampling theory of encoding variability. In A. W. Melton \& E. Martin (Eds.), Coding processes in human memory (pp. 85-123). Washington, DC: Winston.

Brown, G. D. A., Preece, T., \& Hulme, C. (2000). Oscillator-based memory for serial order. Psychological Review, 107, 127-181.

DonaldSON, W. (1996). The role of decision processes in remembering and knowing. Memory \& Cognition, 24, 523-533.

Estes, W. K. (1955). Statistical theory of spontaneous recovery and regression. Psychological Review, 62, 145-154.

FleXser, A. J., \& Bower, G. H. (1974). How frequency affects recency judgments: A model for recency discrimination. Journal of Experimental Psychology, 103, 706-716.

Friedman, W. J. (1993). Memory for the time of past events. Psychological Bulletin, 113, 44-66.

HaRt ley, D. (1834). Observations on man, his fame, his duty, and his expectations (6th ed.). London: Tegg.

HinRICHS, J. V. (1970). A two-process memory-strength theory for judgment of recency. Psychological Review, 77, 223-233.

HinRICHS, J. V., \& BuschKe, H. (1968). Judgment of recency under steady-state conditions. Journal of Experimental Psychology, 78, 574-579.

Hintzman, D. L. (1969). Apparent frequency as a function of frequency and the spacing of repetitions. Journal of Experimental Psychology, 80, 139-145.

Hintzman, D. L. (2001). Judgments of frequency and recency: How they relate to reports of subjective awareness. Journal of Experimental Psychology: Learning, Memory, \& Cognition, 27, 1347-1358.

Hintzman, D. L., Block, R. A., \& Summers, J. J. (1973). Contextual associations and memory for serial position. Journal of Experimental Psychology, 97, 220-229.

Hirshman, E., \& Henzler, A. (1998). The role of decision processes in conscious recollection. Psychological Science, 9, 62-65.

Hirshman, E., \& Master, S. (1997). Modeling the conscious correlates of recognition memory: Reflections on the remember-know paradigm. Memory \& Cognition, 25, 345-351.

Hooke, R. (1969). The posthumous works of Robert Hooke: With a new introduction by Richard S. Westfall. New York: Johnson. (Original work published 1705)

Huppert, F. A., \& Piercy, M. (1976). Recognition memory in amnesic patients: Effect of temporal context and familiarity of material. Cortex, 12, 3-20.

Inoue, C., \& Bellezza, F. S. (1998). The detection model of recognition using know and remember judgments. Memory \& Cognition, 26, 299-308.

JAMES, W. (1890). The principles of psychology (Vol. 1). New York: Holt.

KoffKa, K. (1935). Principles of Gestalt psychology. New York: Harcourt, Brace \& World.

KöHLER, W. (1929). Gestalt psychology. New York: Liveright.

KonORSKI, J. (1961). The physiological approach to the problem of recent memory. In J. F. Delafresnaye (Ed.), Brain mechanisms and learning (pp. 115-132). Springfield, IL: Thomas.

Mayes, A. R., Baddeley, A. D., Cockburn, J., Meudell, P. R., PickERING, A., \& WiLson, B. (1989). Why are amnesic judgments of recency and frequency made in a qualitatively different way from those of normal people? Cortex, 25, 479-488.

Murdock, B. B., JR. (1974). Human memory: Theory and data. Potomac, MD: Erlbaum.

Peterson, L. R. (1967). Search and judgment in memory. In B. Kleinmuntz (Ed.), Concepts and the structure of memory (pp. 153-180). New York: Wiley.

Peterson, L. R., Johnson, S. T., \& Coatney, R. (1969). The effect of repeated occurrences on judgments of recency. Journal of Verbal Learning \& Verbal Behavior, 8, 591-596.

Rosenthal, R., \& Rosnow, R. L. (1985). Contrast analysis. Cambridge: Cambridge University Press.

Shepard, R. N., \& Teghtsoonian, M. (1961). Retention of information under conditions approaching a steady state. Journal of Experimental Psychology, 62, 302-309. 
Wickelgren, W. A. (1974). Single-trace fragility theory of memory dynamics. Memory \& Cognition, 2, 775-780.

XIANG, J.-Z., \& Brown, M. W. (1998). Differential neural encoding of novelty, familiarity and recency in regions of the anterior temporal lobe. Neuropharmacology, 37, 657-676.

Yntema, D. B., \& Trask, F. P. (1963). Recall as a search process. Journal of Verbal Learning \& Verbal Behavior, 2, 65-74.

\section{NOTES}

1. In the case of certain amnesics, this observation may not hold (see Huppert \& Piercy, 1976; Mayes et al., 1989).
2. To quote Hartley (1834), "If the specific nature of memory consists in the great vigour of ideas, and their associations, then, as this vigour abates, it ought to suggest to us a length of time elapsed; and vice versa, if it be kept up, the distance of time ought to appear contracted. Now this last is the case: for the death of a friend, or any interesting event, often recollected and related, appears to have happened but yesterday, as we term it" (pp. 237-238).

3 . For the 1 st subject, the list length was 500 . This subject's data were included in the analysis.

(Manuscript received April 20, 2001; accepted for publication July 21, 2001.) 